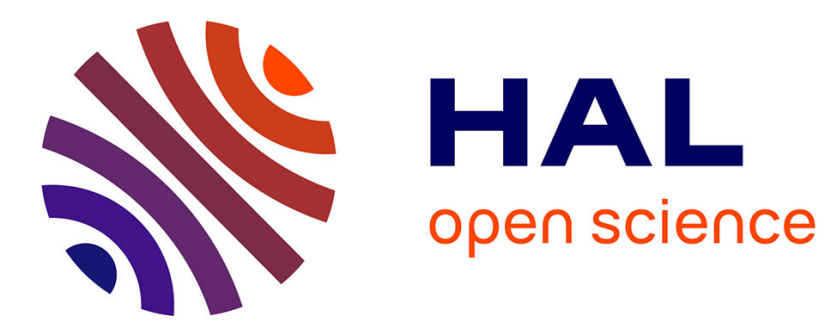

\title{
Driving mechanism for damping and g-factor in non-amorphous ferromagnetic CoFeZr ultrathin films
}

C. Le Graët, D. Spenato, N. Beaulieu, D. Dekadjevi, J-Ph. Jay, S. Pogossian, Bénédicte Warot-Fonrose, J. Ben Youssef

\section{- To cite this version:}

C. Le Graët, D. Spenato, N. Beaulieu, D. Dekadjevi, J-Ph. Jay, et al.. Driving mechanism for damping and g-factor in non-amorphous ferromagnetic CoFeZr ultrathin films. EPL - Europhysics Letters, 2016, 115 (1), pp.17002 - 17002. 10.1209/0295-5075/115/17002 . hal-01707117

\section{HAL Id: hal-01707117 https://hal.science/hal-01707117}

Submitted on 13 Feb 2018

HAL is a multi-disciplinary open access archive for the deposit and dissemination of scientific research documents, whether they are published or not. The documents may come from teaching and research institutions in France or abroad, or from public or private research centers.
L'archive ouverte pluridisciplinaire HAL, est destinée au dépôt et à la diffusion de documents scientifiques de niveau recherche, publiés ou non, émanant des établissements d'enseignement et de recherche français ou étrangers, des laboratoires publics ou privés. 


\title{
Driving mechanism for damping and g-factor in non-amorphous ferromagnetic CoFeZr ultrathin films
}

\author{
C. Le Graët ${ }^{1}$, D. Spenato ${ }^{1(a)}$, N. Beaulieu ${ }^{1}$, D. T. Dekadjevi ${ }^{1}$, J-Ph. Jay ${ }^{1}$, S. P. Pogossian ${ }^{1}$, \\ B. Warot-Fonrose ${ }^{2}$ and J. Ben Youssef ${ }^{1}$ \\ 1 Laboratoire de Magnétisme de Bretagne, CNRS, Université de Bretagne Occidentale - 6 avenue le Gorgeu, \\ 29285 Brest Cedex, France \\ 2 CEMES-CNRS - 29 rue Jeanne Marvig, 31055 Toulouse, France
}

received 10 May 2016; accepted in final form 1 July 2016

published online 27 July 2016

PACS 76.50.+g - Ferromagnetic, antiferromagnetic, and ferromagnetic resonances; spin-wave resonance

PACS 75.70.-i - Magnetic properties of thin films, surfaces, and interfaces

PACS $75.50 . \mathrm{Bb}-\mathrm{Fe}$ and its alloys

\begin{abstract}
We demonstrate that an in-plane uniaxial anisotropy may be induced in nonamorphous soft CoFeZr films. We used broadband ferromagnetic resonance spectroscopy and complex permeability spectra to investigate the spin dynamics in CoFeZr films. We report a systematic study of the FM thickness on the fundamental dynamic parameters such as the effective magnetisation, the $g$-factor and relaxation mechanisms. Our study reveals that the decrease of the effective magnetisation mesured with FMR with thickness is not due to perpendicular anisotropy but to low dimentionality. Moreover, we observed a decrease of the $g$-factor with thickness and a modification of the ratio of the orbital to the spin magnetic moment. These films exhibit good high-frequency performance red (i.e. high permeability in a broad frequency range and a low damping) at low thickness of about a few nanometers.
\end{abstract}

Copyright (C) EPLA, 2016

Nowadays, spintronics devices and magnetic media have to operate at the gigahertz regime (i.e. with ns reversal times). Magnetic thin films are widely used and studied because the ability of their magnetization to precess/reverse in a high frequency-short time range (several GHz/ns) [1,2]. For high-frequency applications, these magnetic materials need to have a large permeability in a broad frequency range [3]. The key parameters that govern spin dynamics are the saturation magnetization, the effective field and the (Lande) factor [4].

FeCo alloy should be, at first glance, one of the most competitive candidates because of its high saturation magnetization $M_{S}(2.45 \mathrm{~T})$. However, as-deposited FeCo films exhibit high coercive field ranging from 100 to 200 Oe, inplane isotropic magnetic anisotropy and a large magnetocrystalline anisotropy which hinders the high-frequency applications ([5] and references therein). A solution is to produce amorphous CoFe-based alloys by alloying metalloid into the FM matrix: the addition of a metalloid in the ferromagnet (FM) destroys the cristallinity,

\footnotetext{
(a) E-mail: david.spenato@univ-brest.fr
}

reduces the saturation magnetization and the coercivity. In addition, it is possible to induce a uniaxial magnetic anisotropy (UMA) in such thin films [6]. Consequently, in the last two decades many studies have focused on amorphous thin ferromagnetic films such as $\mathrm{CoFeX}(\mathrm{X}=\mathrm{Zr}[7]$, Hf $[8], \mathrm{B}[6] \ldots)$. These alloys are very attractive for high-frequency applications because of their large magnetization $\left(M_{S}\right)$ (due to the presence of $\mathrm{CoFe}$ ), their large UMA, their relative softness (i.e., low coercitive field $H_{c}$ ) and their high resistivity (to avoid eddy current losses) [3,9]. Moreover, they are also promising candidates either for electrode in magnetic tunnel junctions (MTJs) [10,11], for current-driven switching at low current densities or for hybrid magnetic-ferroelectric systems [12]. Apart from high $M_{S}$ and low $H_{c}$, damping is an interesting problem because it determines in the free layer the critical switching current in spin-torque-transfert (STT) bases devices $[13,14]$ and then the power consumption in STT based MRAM.

Despite the great technological importance of CoFebased alloys, many fundamental questions about their magnetic properties remain open such as the origin of the 
UMA in such films [15]. In low-dimensional systems (i.e., thin films, nano wires and nano dots) interfaces play a major role, and understanding the effect of the interface on the driving parameters for the magnetization processes is crucial for both fundamental physics and engineering. For example, the broken symmetry of an interface has long been known to change the orbital and spin moments at the interface compared to the bulk region (see, for example, [16] and [17] and references therein). Moreover, dynamic studies for various frequencies and low thickness range for CoFe alloys is still missing: most of the studies deal with rather thick films (several tens of $\mathrm{nm}[18,19]$ ), focus on $\mathrm{CoFeB}$ and very few alternative amorphous alloys are studied. There is a lot of dispersion in the report dynamic properties, thus a detailed knowledge about such properties in these polymorphic structures is of essential importance to address this lack.

In this article we show that an in-plane magnetic anisotropy (UMA) and magnetic softness may be induced in crystalline $\mathrm{CoFeZr} / \mathrm{Al}_{2} \mathrm{O}_{3}$ bilayers, commonly assumed to be inherent to amorphous phase of such alloys. We report an systematic study of the FM thickness on the fundamental dynamic parameters such as the $g$-factor and relaxation mechanisms. Indeed, we reveal that the low dimensionality drives the magnetization properties.

$\mathrm{Co}_{28} \mathrm{Fe}_{65} \mathrm{Zr}_{7}\left(t_{F M}\right) / \mathrm{Al}_{2} \mathrm{O}_{3}(5 \mathrm{~nm})$ bilayers where grown by standard RF diode sputtering onto Si (111) substrates. A CoFe target with $\mathrm{Zr}$ chips was used and the composition was checked with Electron Probe Micro Analysis (EPMA) measurements. The base pressure prior to the film deposition was typically $10^{-7} \mathrm{mbar}$. The FM CoFeZr thicknesses were $t_{F M}=3,3.5,4,5,6,7,11,16$ and $20 \mathrm{~nm}$. An in-plane magnetic field of $2.4 \mathrm{kA} / \mathrm{m}$ was applied, during deposition, to induce a UMA. Structural analysis were performed by TEM experiments on cross-sectional lamellas, thinned by mechanical polishing and argon ion milling at low temperature using a Gatan Precision Ion Polishing System equipped with a liquid nitrogen cooling system. The samples were examined in high resolution electron microscopy (HREM) on a TECNAI F-20 operating at $200 \mathrm{kV}$, equipped with a spherical aberration corrector to avoid the delocalization effect at interfaces and to achieve a $0.12 \mathrm{~nm}$ resolution. Static magnetic measurements where made with a vibration sample magnometer (VSM).

Typical magnetization curves of as-deposited CoFeZr films on $\mathrm{Si}$ (111) substrate are shown in the inset of fig. 1(a). The angle between the in-plane magnetic field and the deposition field direction may be tuned during measurement. On this figure it is clear that an UMA in the film is induced and that the easy axis is parallel to the deposition field direction. Besides, the addition of $\mathrm{Zr}$ have successfully changed the magnetocristalline anisotropy generally observed in the FeCo. This has been observed on our samples whatever the FM thickness s. In order to go deeper, we have checked the micro-structure through HRTEM (fig. 1(c)). For the thinner sample (3 nm)
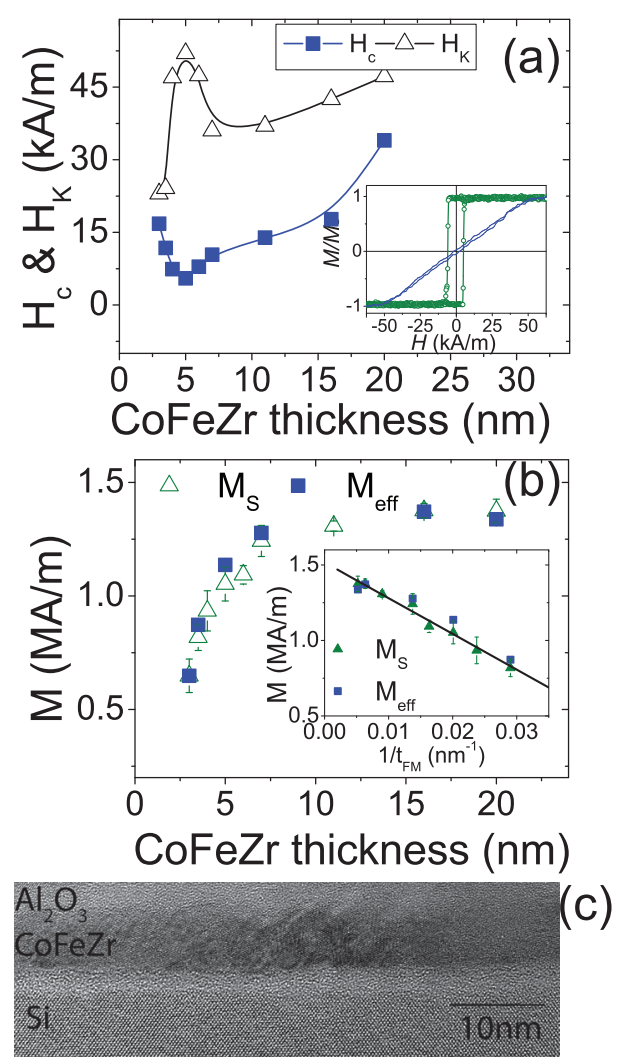

Fig. 1: (Colour online) (a) Thickness dependence of coercive $\left(H_{c}\right)$ and anisotropy $\left(H_{k}\right)$ fields with CoFeZr thickness. Solid lines are to guide the eyes. Inset of (a): a typical hysteresis loop of the $\mathrm{CoZrFe} / \mathrm{Al}_{2} \mathrm{O}_{3}$ bilayers measured along the easy axis (parallel to the deposition field direction) and in a perpendicular direction, showing a well-defined in-plane anisotropy. (b) Saturation magnetization $M_{S}$ and the effective magnetization $M_{\text {eff }}$ as a function of thickness of CoZrFe in $\mathrm{CoZrFe} / \mathrm{Al}_{2} \mathrm{O}_{3}$ bilayers. Inset of (b): plot of $M_{S}$ and $M_{\text {eff }}$ as a function of reciprocal thickness. The linear fit is used to highlight the interface contribution. (c) High-resolution TEM image of a typical $\mathrm{CoZrFe}(7 \mathrm{~nm}) / \mathrm{Al}_{2} \mathrm{O}_{3}$ bilayer.

the HTREM reveals (not shown) amorphous and crystallized parts. One should notice that the roughness is less than $1 \mathrm{~nm}$. Surprisingly, the HRTEM shows a polycrystalline structure of the CoFeZr layer for all the other thickness 5 to $20 \mathrm{~nm}$. Indeed it demonstrates that UMA and magnetic softness (low coercivity) may be induced in polycristalline $\mathrm{CoFeZr}$ alloys, generally considered proper to amorphous alloys in such composition [7,20].

In fig. 1(a) we show the thickness dependence of both coercive and anisotropy field. Quantitatively, our samples present good softness (coercive about a 1 to $3 \mathrm{kA} / \mathrm{m}$ ) and UMA field that lies in the range 20 to $40 \mathrm{kA} / \mathrm{m}$.

The magnetization dynamics was probed with two broadband techniques on a selection of samples (i.e., 3, $5,6,7,16$ and $20 \mathrm{~nm}$ ). The first one is ferromagnetic resonance (FMR) which was investigated within a range of microwave frequencies between 3 and $40 \mathrm{GHz}$ using a wideband resonance spectrometer with a non-resonant 
micro-strip transmission line [21]. The FMR is measured via the derivative of the microwave power absorption $(\mathrm{d} P / \mathrm{d} H)$ using a small rf exciting field. Resonance spectra were recorded with the applied static magnetic field oriented in-plane at an angle of 90 degrees relative to the depositing field. The second technique is the complex permeability frequency spectra (CPS) measured from $30 \mathrm{MHz}$ to $3 \mathrm{GHz}$ on samples with thickness from 7 to $20 \mathrm{~nm}$ using a broadband method based on the measurement of the reflection coefficient $S_{11}$ of a single-turn coil loaded by the film under test with a network analyser. In such coil, for the frequency band used, we consider that the electromagnetic field is propagating in a transverse electromagnetic (TEM) mode. When the film is in the coil, because of the small magnetic volume, the perturbation of the EM field by the magnetic film is small and the EM field can still be considered as TEM (called quasi-TEM approximation [22].

In FMR, the measured resonance frequency as a function of resonance field is linear when the anisotropy contributions are much smaller than the applied magnetic field and it allows to determine the $g$-value and the effective magnetization $\left(M_{\text {eff }}\right)$. Since surface anisotropies may exist (due to the broken symmetry of the interface) and be substantial for very thin samples, the resonance condition in the perpendicular configuration (i.e., the applied magnetic field perpendicular to the film plane) is [23]

$$
\begin{aligned}
\frac{\omega_{r e s}}{\mu_{0} \gamma} & =H_{r e s}-M_{e f f} \\
\left(M_{e f f}\right. & \left.=M_{s}-\frac{2 K_{\perp}}{\mu_{0} M_{s} t_{F M}}\right),
\end{aligned}
$$

where $H_{\text {res }}$ is the resonance field, $\gamma=\left(g \mu_{B}\right) / \hbar$ is the gyromagnetic ratio, $K_{\perp}$ is the perpendicular magnetic anisotropy coefficient and $\hbar$ is the reduced Planck's constant. Measurement of $M_{\text {eff }}$ and $g$ is straightforwardly achieved by measurements of the resonance field $H_{\text {res }}$ for several frequencies and using eq. (1). The evolution of $M_{\text {eff }}$ vs. the FM thickness is presented in fig. 1(b). The observed decrease of $M_{\text {eff }}$ is generally observed in ultrathin films and is often explained by the existence of a surface anisotropy term, consisting of the different interface contribution $[17,23]$. Indeed, this value has to be compared with the one obtained from static measurements (i.e., $M_{S}$ ). A plot of the saturation magnetization $M_{S}$ is also presented in fig. 1(b). Obviously, the measured values of both $M_{s}$ and $M_{\text {eff }}$ are almost identical, whatever the FM thickness. It means that the surface anisotropy is negligeable. Thus, the reduction of the effective magnetisation $M_{\text {eff }}$ with thickness is due to a diminution of the saturation magnetisation related to low-dimensionality effect and not to surface anisotropy effect (which one may naively predict with the model above (eq. (1)). Moreover the good $1 / t_{F M}$ dependence of both $M_{\text {eff }}$ and $M_{s}$ (inset of fig. 1(b)) suggest that there is a low-dimensionality dependence in this thickness range.
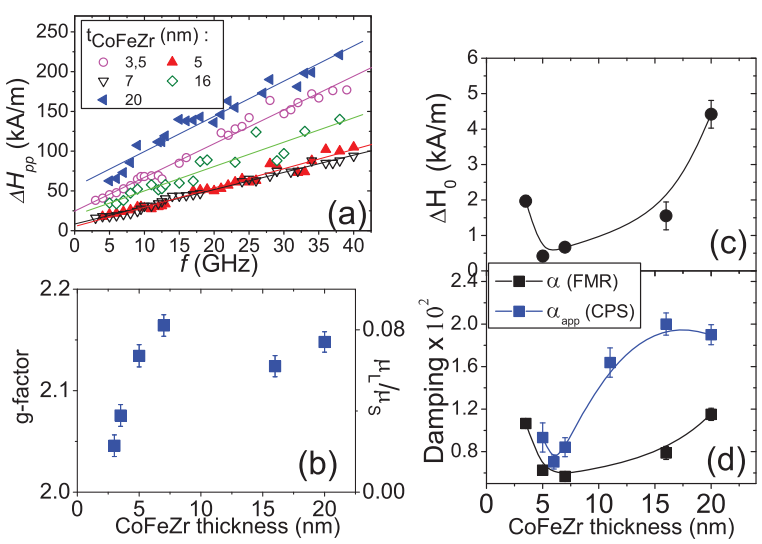

Fig. 2: (Colour online) (a) Frequecy dependence of the peakto-peak linewidth $\Delta H_{p p}$ for several CoFeZr thicknesses and with the external field in the film plane along the hard axis. The solid lines are linear fits according to (3). (b) CoFeZr thickness dependence of both $g$-factor and ratio of the orbital and spin moment $\left(\frac{\mu_{L}}{\mu_{S}}\right)=\frac{g-2}{2}$ according to [24]. (c) Thickness dependence of the extrinsic contribution $\Delta H_{0}$ in CoFeZr. (d) Intrinsic damping parameter deduced from FMR linewidth $(\alpha(\mathrm{FMR}))$ and the apparent damping $\left(\alpha_{\text {app }}(\mathrm{CPS})\right)$ deduced from Complex permeability measurements.

The fundamental quantity which characterizes the magnetic moment is the $g$-factor, it is directly related to the ratio of the orbital and spin momentum $\left.\left(\mu_{L} / \mu_{S}=(g-2) / 2\right)\right)$ according to [24]). In $3 \mathrm{~d}$ transition elements the orbital momentum is quenched by the strong crystalline field and therefore the $g$-factor is close to the value of the free electron (i.e., $g=2$ ). Nevertheless the $g$-factor is known to depend on composition (for example in FeCo alloys [25]) and to be anisotropic $[17,26]$. Moreover, $g$ is strongly influenced by surface and interface effects as it depends on the local symmetry. Such interface effects may lead to strong enhancements of $\mu_{L} / \mu_{S}$ ratios (for example in $\mathrm{Fe} / \mathrm{V}$ superlattices [16]) or to a decrease with an $1 / t$ law of the $g$-factor [17]. These effects are often related to geometric the confinement in the samples that causes a perturbation of the electron orbits at the interface or hybridization $[17,27]$.

The values of the $g$-factor (extracted from FMR data), plotted as a function of the FM thickness is given in fig. 2(b). The $g$-factor decreases with decreasing thickness, consistent with previous studies in metallic thin films $[17,23]$. For the larger thickness, the value of the $g$-factor 2.15, which lies in the range of the bulk values of Fe (2.09) and fcc Co (2.15) [24,25]. Recent studies have mentioned values from 2.13 in CoFeZr/AlZr/CoFeZr [28] and values from 2.159 to 2.186 depending on the Co-to-Fe ratio and the cristalinity of $1 \mathrm{~nm}$ thick CoFeB films [29]. Nevertheless our study reveals that the $g$-factor decreases as the thickness decreases to reach 2.04. This result shows that in CoFeZr we observe $g$-factor values which is pretty close from the free electron value indicating a small spin-orbit interaction as observed in Heusler alloys $\mathrm{NiMnSb}$ [30]. Since the $g$-factor describes the ratio of the 
orbital to spin moments (see above), it is interesting to plot the values of $\mu_{L} / \mu_{S}$ vs. the FM thickness, the results are presented in fig. 2(a). In amorphous films, due to the lack of long-range ordering of atomic positions, one would expect that the degree of quenching of orbital momentum is quite low, leading to a high $g$-factor value. However, the $g$-factor determined here indicates that the orbital moment might still be partially quenched due to the polycrystalline state of our samples. Moreover, the $\mu_{L}$-to- $\mu_{S}$ ratio decreases from 0.08 to 0.02 with decreasing thickness. It should be noted that a recent study demonstrated that, in the amorphous state of CoFeZr [31], the $\mu_{L} / \mu_{S}$ diminishes for both Fe and Co when the CoFeZr layer is decreased in thickness. It should be noted that a reduction of the magnetization we observed cannot be explained by the orbital moment. Indeed, the contribution from the hybridisation of the ferromagnetic layer at the interface with the non magnetic layer should be important in these effects. However more complementary experiments are necessary (such as X-ray circular dichroism) to determine the specific contribution of both Co and Fe to these magnetic properties.

The other fundamental quantity that characterizes the high-frequency response of a ferromagnetic material is the damping, which is usually taken into account by a phenomenological term in the well-known equation of motion for the magnetization $M$ in an effective field $H_{\text {eff }}$ [32]:

$$
\frac{\mathrm{d} \mathbf{M}}{\mathrm{d} t}=-\mu_{0} \gamma\left(\mathbf{M} \times \mathbf{H}_{e f f}\right)+\frac{1}{M} \mathbf{M} \times \alpha \frac{\mathrm{d} \mathbf{M}}{\mathrm{d} t} .
$$

In eq. (2), $\gamma$ is the gyromagnetic ratio, and $\alpha$ is the mean Gilbert phenomenological damping parameter.

In the parallel FMR configuration, with the magnetization parallel to the applied field, the time derivative $\mathrm{d} \mathbf{M} / \mathrm{d} t$ Gilbert term in the equation of motion produces a FMR linewidth linear with the microwave frequency $\mathbf{f}$. However, in many magnetic systems, while a linear behaviour is observed, the linewidth fails to extrapolate to zero with vanishing frequency. This zero-frequency contribution $\Delta H_{0}$ reflects the effect of magnetic inhomogeneity on the linewidth. Thus, frequency dependent studies provide intrinsic and extrinsic $\left(\Delta H_{0}\right)$ contributions to the relaxation. The field-swept linewidth, in a given direction, may be written as [33]

$$
\Delta H_{P P}=\Delta H_{0}+\frac{2}{\sqrt{3}} \frac{\alpha}{\mu_{0} \gamma} 2 \pi f,
$$

where $\Delta H_{0}$ is the inhomogeneous broadening. The second term is the Gilbert contribution that represent intrinsic contribution. One should note that non-linear behaviour may be observed and attributed to two-magnon scattering ([34] and references therein). It is not effective when the magnetic field is applied perpendicular to the film plane, but may be important for the other direction [35].

As observed in fig. 2(a), the FMR linewidth, $\Delta H_{P P}$, is linearly dependent on the microwave frequency, whatever the FM thickness is. For higher frequencies non-linear

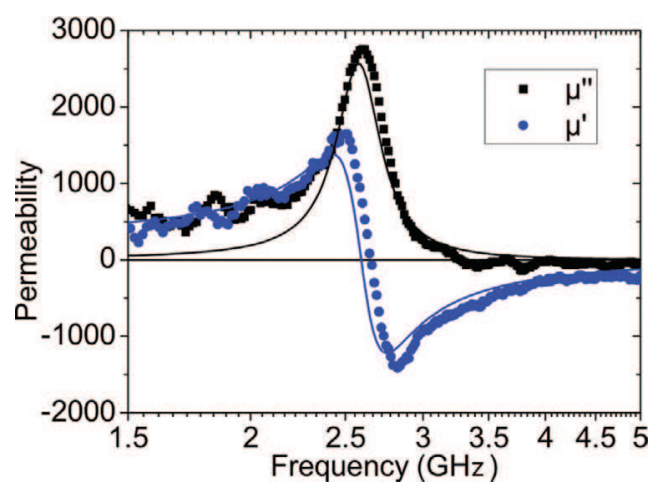

Fig. 3: (Colour online) Real and imaginary par of the complex permeability spectra measured along a direction perpendicular to the easy axis of the CoFeZr layer $(6 \mathrm{~nm})$ and calculated using eq. (5) in [42].

behavior could be observed du to two magnon processes $[35,36]$. We have not observed such non-linear behavior over our entire experimental frequency range. This result suggest that two-magnon scatering is not significant in our thickness range (from 3.5 to $20 \mathrm{~nm}$ ), but could be significant at lower thickness as observed in $\mathrm{CoFeB}$ free layers in $\mathrm{MgO}$-based magnetic tunnel junction [37].

From these data, we have extracted both extrinsic (fig. 2(c)) and intrinsic ((fig. 2(d)) contribution to the FMR linewith for different FM thickness s. On these figures, is is clear that a broad valley with a minimum at around a FM thickness of about $6 \mathrm{~nm}$ is observed. Before this thickness, the intrinsic damping parameter decreases from $\alpha=0.01064$ at $3 \mathrm{~nm}$ to 0.0056 at $7 \mathrm{~nm}$. Such a decrease of the damping parameter has been observed in this thickness range (from 1 to $7 \mathrm{~nm}$ ) on NM/FM and the authors have explained it in terms of two magnon scattering and spin pumping [38]. This argument does not support our data since this effect deals with normal metal (NM) adjacent layers and our interfaces are $\mathrm{Si} / \mathrm{FM} / \mathrm{Al}_{2} \mathrm{O}_{3}$. Above a thickness of $7 \mathrm{~nm}$, both contributions to the relaxation increase with increasing thickness up to $\alpha=0.0111$ at $20 \mathrm{~nm}$. Recent studies on $\mathrm{CoFeB}$ amorphous alloys [37,38] show a monotonic decrease of the Gilbert damping parameter with the FM thickness attributed to partially spin-pumping effect and other non local effects [37]. Moreover, the Gilbert damping constant may be strongly correlated to the $g$-factor and the magnetization. Indeed it should scale like $(g-2)^{2}$ [39] as was very recently observed in FeCoB ultrathin films [29]. We have not observed such variation in our samples. Nevertheless, our results suggest that the damping can be tuned by varying the FM film thickness. The lowest value for $\alpha$ is 0.0056 . These are typical values for low loss metal films [4,23] and amorphous films [29]. Such a small value of $\alpha$ is advantageous for reducing spin torque critical current in MTJS for example.

Finally we present in fig. 3 a typical complex permeability spectra (CPS) of a CoFeZr with thickness $6 \mathrm{~nm}$, where $\mu^{\prime}$ and $\mu^{\prime \prime}$ represent respectively the real and imaginary 
part of the complex permeability measured perpendicularly to the easy axis. The value of the real part of the permeability $\mu^{\prime}$ is about 300 for a resonance frequency of $2.5 \mathrm{GHz}$. Such a value of $\mu^{\prime}$ has not been obtained, as far as we know, for such low thickness. Moreover, these values are similar to the one obtained much thicker (about $100 \mathrm{~nm}$ ) FeCo alloys such as FeCoSiN [40] or FeCoTaN [9] or $\mathrm{FeCoB}$ [41].

The zero-field apparent damping parameter $\alpha_{a p p}$ may also be measured from a fit of the complex permeability spectra with analytical formula of the permeability tensor terms [42]. In the fits, the values of $M_{S}$ and $H_{k}$ have been taken from static measurements. It should noted that during the measurement of the CPS, no static magnetic field is applied. Consequently, the "zero-field" apparent damping parameter $\alpha_{a p p}$ contains extrinsic contributions and some inhomogeneous frequency dependent line-width since the measurement is made in a non-saturated magnetic state $[43,44]$. The results are presented in fig. 2(d). Both the intrinsic damping parametrer measured by FMR $\alpha$ and the apparent damping parameter $\alpha_{a p p}$ measured by CPS have the same thickness dependence. Because $\alpha_{a p p}$ contains inhomogenous broadening, its value remains higher than the intrinsic one, $\alpha$.

For lowest FM thickness (below $7 \mathrm{~nm}$ ), the values of the apparent damping parameters are close to the one obtained from FMR. This suggest that at low thickness the contribution to the damping of the inhomogenous broadening is not significant. For the highest thickness (above $7 \mathrm{~nm}$ ), the values of the zero-field damping parameter are much higher than the intrinsic damping parameter extracted from FMR due to a much more significant contribution of inhomogenous broadening. The lowest value of $\alpha_{\text {app }}$ measured by CPS is 0.00703 at $6 \mathrm{~nm}$. This value is lower than the values measured with CPS on some FeCo alloys such as FeCoTaN (0.015) [9]. On the other hand, it is close to the one obtained on FeCoB (0.008) [45] which is known to be a material with low damping interesting for applications in MTJs.

To conclude, we have successfully induced a UMA and magnetic softness in polycrystalline CoFeZr bilayers, demonstrating that they are not proper to the amorphous state of these alloys as has been considered for many decades. Our study reveals that the decrease of the effective magnetisation mesured with FMR is not due to perpendicular anisotropy but to low dimentionality. Moreover, we observed a decrease of the $g$-factor with thickness and a modification of the ratio of the orbital to the spin magnetic moment. For low thickness the $g$-factor decreases to 2.04 indicating very small spin-orbit coupling. Moreover, relaxation mechanisms are studied, showing nontrivial variation of the intrinsic damping parameter with the FM thickness. Finally, because of their large magnetization $\left(M_{S}\right)$, their large UMA, their relative softness (i.e., low coercitive field $H_{c}$ ), our films exhibit very good high-frequency performance (i.e., high permeability in a broad frequency range and a low damping) even for thickness of about $6 \mathrm{~nm}$. Indeed, because of their magnetostrictive properties, CoFeZr films may be used in Hybrid FM/Piezoelectric composite and should provide great opportunities for electric-field-controlled, low consumption energy, tunable microwave devices [12].

This work was partly supported by Region Bretagne ARED (No. 3596).

\section{REFERENCES}

[1] Katine J. A., Albert F. J., Buhrman R. A., Myers E. B. and Ralph D. C., Phys. Rev. Lett., 84 (2000) 3149.

[2] Kubota H., Fukushima A., Yakushiji K., Nagahama T., Yuasa S., Ando K., Maehara H., Nagamine Y., Tsunekawa K., Djayaprawira D. D., Watanabe N. and Suzuki Y., Nat. Phys., 4 (2008) 37.

[3] Wang S. X., Sun N. X., Yamaguchi M. and Yabukami S., Nature, 407 (2000) 150.

[4] Beach G., Silva T., Parker F. and Berkowitz A., IEEE. Trans. Magn., 39 (2003) 2669.

[5] Wu Y., Han G.-C. and Kong L., J. Magn. \& Magn. Mater., 322 (2010) 3223.

[6] Hindmarch A. T., Kinane C. J., MacKenzie M., Chapman J. N., Henini M., Taylor D., Arena D. A., Dvorak J., Hickey B. J. and Marrows C. H., Phys. Rev. Lett., 100 (2008) 117201.

[7] Suran G., Naili M., Niedoba N., Machizaud F., Acher O. and Pain D., J. Magn. E Magn. Mater., 192 (1999) 443.

[8] Li S., Zhigao H., Duh J.-G. and Yamaguchi M., Appl. Phys. Lett., 92 (2008) 092501.

[9] Bekker V., Seemann K. and Leiste H., J. Magn. \& Magn. Mater., 296 (2006) 37.

[10] Paluskar P. V., Attema J. J., de Wijs G. A., Fiddy S., Snoeck E., Kohlhepp J. T., Swagten H. J. M., de Groot R. A. and Koopmans B., Phys. Rev. Lett., 100 (2008) 057205.

[11] Luo Y., Esseling M., Käufler A., SAmwer K., Dimopoulos T., Gieres G., Vieth M., Rührig M., Wecker J., Rudolf C., Niermann T. and Seibt M., Phys. Rev. B, 72 (2005) 014426.

[12] Sun N. X. and Srinivasan G., SPIN, 02 (2012) 1240004.

[13] Slonczewski J., J. Magn. 63 Magn. Mater., 159 (1996) L1.

[14] Herzer G., IEEE Trans. Magn., 26 (1990) 1397.

[15] Hindmarch A. T., Rushforth A. W., Campion R. P., Marrows C. H. and Gallagher B. L., Phys. Rev. B, 83 (2011) 212404.

[16] Anisimov A. N., Farle M., Poulopoulos P., Platow W., Baberschke K., Isberg P., WÄppling R., Niklasson A. M. N. and Eriksson O., Phys. Rev. Lett., 82 (1999) 2390.

[17] Shaw J. M., Nembach H. T. and Silva T. J., Phys. Rev. B, 87 (2013) 054416.

[18] Li S., Huang Z., Duh J.-G. and Yamaguchi M., Appl. Phys. Lett., 92 (2003) 092501.

[19] Li S., Huang Z., Duh J.-G. and Yamaguchi M., J. Phys. D: Appl. Phys., 41 (2008) 055004. 
[20] Lidbaum H., Raanaei H., Papaioannou E. T., Leifer K. and HJörvarsson B., J. Cryst. Growth, 312 (2010) 580 .

[21] Grä̈t C. L., Spenato D., Pogossian S., Dekadjevi D. and Youssef J. B., Appl. Phys. Lett., 94 (2009) 262502.

[22] Pain D., Ledieu M., Acher O., Adenot L. and Duverger F., J. Appl. Phys., 85 (1999) 5151.

[23] Beaujour J.-M., Ravelosona D., Tudosa I., Fullerton E. E. and Kent A. D., Phys. Rev. B, 80 (2009) 180415; Nibarger J., Lopusnik R., Celinski Z. and Silva T., Appl. Phys. Lett., 83 (2003) 93.

[24] Meyer A. and Asch G., J. Appl. Phys., 32 (1961) S330.

[25] Schreiber F., Pflaum J., Frait Z., Mühge T. and Pelzl J., Solid State Commun., 93 (1995) 965.

[26] Platow W., Anisimov A., Dunifer G., Farle M. and Baberschke K., Phys. Rev. B, 58 (1998) 5611.

[27] Nakajima N., Koide T., Shidara T., Miyauchi H., Fukutani H., Fujimori A., Iio K., Katayama T., NÝvlt M. and Suzuki Y., Phys. Rev. Lett., 81 (1998) 5229.

[28] Fu Y., Barsukov I., Raanaei H., Spasova M., Lindner J., Meckenstock R., Farle M. and HJörvarsson B., J. Appl. Phys., 109 (2011) 113908.

[29] Devolder T., Ducrot P.-H., Adam J.-P., Barisic I., Vernier N., Kim J.-V., Ockert B. and Ravelosona D., Appl. Phys. Lett., 102 (2013) 022407.

[30] Heinrich B., Wolterdorf G., Urban R., Mosendz O., Schmidt G., Bach P., Molenkamp L. and Rozenberg E., J. Appl. Phys., 95 (2004) 7462.

[31] Hase T., Raanaei H., Lidbaum H., Sánchez-Hanke C., Wilkins S., Leifer K. and Huörvarsson B., Phys. Rev. B, 80 (2009) 134402.
[32] Gilbert T., IEEE Trans. Magn., 40 (2004) 3443.

[33] Urban R., Heinrich B., Woltersdorf G., Ajdari K., Myrtle K., Cochran J. and Rozenberg E., Phys. Rev. B, 65 (2002) 020402.

[34] Arias R. and Mills D., Phys. Rev. B, 60 (1999) 7395.

[35] Lenz K., Wende H., Kuch W., Baberschke K., Nagy K. and JÁnossy A., Phys. Rev. B, 73 (2006) 144424.

[36] Twisselmann D. and McMichael R., J. Appl. Phys., 93 (2003) 6903.

[37] liu X., Zhang W., Carter M. and Xiao G., J. Appl. Phys., 110 (2011) 033910.

[38] Tserkovnyak Y. and Bratas A., Phys. Rev. Lett., 88 (2002) 117601; Beaujour J.-M., Lee J., Kent A., Krycka K. and KaO C.-C., Phys. Rev. B, 74 (2006) 214405; Natarajarathinam A., Tadisina Z. R., Mewes T., Watts S., Chen E. and Gupta S., J. Appl. Phys., 112 (2012) 053909.

[39] Kamberskỳ V., Can. J. Phys., 48 (1970) 2906.

[40] Feng X., Phuoc N. N., Zhang X., Ma Y., Chen X. and Ong C. K., J. Appl. Phys., 104 (2008) 093903.

[41] Zhang B. S., Yuan Z., ZhaO H., Yang Y. and Tang D. M., IEEE Trans. Magn., 52 (2016) 1.

[42] Spenato D., Fessant A., Gieraltowski J., Loaec J. and Gall H. L., J. Phys. D: Appl. Phys., 26 (1993) 1736.

[43] Nibarger J. P., Lopusnik R. and Silva T. J., Appl. Phys. Lett., 82 (2003) 2112.

[44] Kuanr B., Camley R. E. and Celinski Z., Appl. Phys. Lett., 87 (2005) 012502.

[45] Bilzer C., Devolder T., Kim J.-V., Counil G., Chappert C., Cardoso S. and Freitas P. P., J. Appl. Phys., 100 (2006) 053903. 